\title{
Technique to Analyze Characteristics of Turbomachinery Broadband Noise Sources
}

\author{
W. Jürgens, U. Tapken†, B. Pardowitz $\ddagger$ P. Kausche ${ }^{\S}$ \\ German Aerospace Center (DLR), Institute of Propulsion Technology, Berlin, Germany \\ G. J. Bennett \\ Trinity College Dublin, Ireland \\ L. Enghardt" \\ German Aerospace Center (DLR), Institute of Propulsion Technology, Berlin, Germany
}

\begin{abstract}
In this paper an experimental technique for the decomposition of broadband ducted sound fields into their mode constituents is applied to two laboratory scale experimental setups. The broadband mode analysis technique is based on spectral coherences. In both cases, incoherent broadband sound fields were excited by means of a loudspeaker array. The results derived from the basic setup S1 were validated successfully with analytical findings. The second setup S2 mimics the fundamental arrangement in an aero-engine exhaust, consisting of a broadband sound source (an annular combustor ring) and a turbomachinery stage (the LP turbine). In between, microphone arrays are arranged to acquire the broadband sound fields. Sample results of setup S2 are presented analyzed by means of the new technique. The experimental outcome proves the usefulness of the analysis technique to help interpreting and understanding broadband sound propagation in turbomachinery flow ducts.
\end{abstract}

\section{Introduction}

Turbomachinery components as fan, compressor, combustor and turbine produce noise of various spectral characteristics. At present, research is focusing on the development of techniques that are suited to analyze the broadband sound field constituents. A technique that can be applied for mode decomposition of broadband ducted sound fields was presented by Enghardt et al. ${ }^{1}$ In addition to the estimate of mode amplitude spectra - being useful e. g. with regard to the quantification of the mean emitted sound power - the method yields explicitly mode coherence functions, i. e. coherences for pair-wise combinations of mode orders. It is the main objectives of the present paper to demonstrate that measured mode coherence functions give insight into the spatial distribution of noise sources and enables the investigation of propagation effects such as mode scattering.

In the first part of the paper (section III) an improved version of Enghardt's method will be validated against theoretical results for a generic test case. The employed broadband-noise analysis method is based on sound pressure cross-correlation measurements within an array of sensors as described in section II.A. The corresponding theory outlined in section II.B assumes monopole sources located in one or two rings driven with completely incoherent signals. In a broader sense the theory extends the two-source examination by Michalke et al. ${ }^{2}$ and the single ring model described by Tester et al. ${ }^{3}$ It will be shown that depending on the number and the distribution of the sources specific mode correlations result.

\footnotetext{
${ }^{*}$ Research Scientist, Engine Acoustics Department, Müller-Breslau-Str. 8, 10623 Berlin, Germany.

${ }^{\dagger}$ Research Scientist, Engine Acoustics Department, Müller-Breslau-Str. 8, 10623 Berlin, Germany, Email: ulf.tapken@dlr.de

$\ddagger$ Junior Scientist, Engine Acoustics Department, Müller-Breslau-Str. 8, 10623 Berlin, Germany.

§Junior Scientist, Engine Acoustics Department, Müller-Breslau-Str. 8, 10623 Berlin, Germany.

ฯ Lecturer, Dept. of Mechanical and Manufacturing Engineering, Trinity College, Dublin 2, Ireland.

" Professor, Engine Acoustics Department, Müller-Breslau-Str. 8, 10623 Berlin, Germany.
} 
In the second part of the paper, the experimental technique is applied to a complex test rig (see section IV). The rig was specifically designed for the simulation of the most relevant acoustic features of a turbo-shaft engine in the low to medium frequency range, i.e. a combustor emits noise that afterwards is scattered by a stator-rotor stage.

\section{Theoretical background}

\section{II.A. Determination of mode coherences}

The sound field within a duct is described as a superposition of an infinite number of modes. Under the constraints of incompressible and isentropic flow, constant axial mean flow profile, stationary mean temperature and density, the solution of the convective Helmholtz equation is given in cylindrical coordinates by a linear superposition of modal terms as follows: ${ }^{4}$

$$
p(x, r, \phi)=\sum_{m=-\infty}^{\infty} \sum_{n=0}^{\infty}\left(A_{m n}^{+} e^{i k_{m n}^{+} x}+A_{m n}^{-} e^{i k_{m n}^{-} x}\right) f_{m n}(r) e^{i m \phi} .
$$

Here $k_{m n}^{+}$and $k_{m n}^{-}$denote the axial wave numbers, $A_{m n}^{+}$and $A_{m n}^{-}$the complex amplitudes of the mode with the azimuthal order $m$ and the radial order $n$ for propagation in and against flow direction, respectively. In case of hard-wall acoustic boundary conditions, the modes form an orthogonal eigensystem. The modal shape factors are given by $f_{m n}(r)=\left(F_{m n}\right)^{-1 / 2}\left(J_{m}\left(\sigma_{m n} r / R\right)+Q_{m n} Y_{m}\left(\sigma_{m n} r / R\right)\right)$, with $J_{m}$ and $Y_{m}$ being the Bessel functions of first and second kind and order $m$ with associated hard-wall cylindrical eigenvalues $\sigma_{m n}$ and $Q_{m n}$. The eigenvalues depend on the hub-to-tip ratio $\eta$. $Q_{m n}$ is zero for non-annular cylinders; $R$ is the outer duct radius. The definition of the normalization factor $F_{m n}$ is given in Ref. ${ }^{5}$

The characteristics of the modes depend on their generation mechanism. In the case of broadband noise, a statistical description of the modes in terms of mean values and spatial cross-correlation is necessary. In what follows, correlations between modes are determined by a technique that originated from a discussion with P. Joseph during a stay of L. Enghardt at the Institute of Sound and Vibration Research (ISVR) in Southampton, UK. The method yields the same results as a previous approach ${ }^{1}$ but the formulation is more straightforward.

Assuming linearity, the vector of pressure measurements $\mathbf{p}$ may be related to the vector of mode amplitudes

$$
\mathbf{a}=\left(\mathbf{a}^{+}, \mathbf{a}^{-}\right)=\left(A_{-M 0}^{+}, A_{-M 1}^{+}, \ldots, A_{00}^{+}, \ldots, A_{M N}^{+}, A_{-M 0}^{-}, A_{-M 1}^{-}, \ldots, A_{00}^{-}, \ldots, A_{M N}^{-}\right)
$$

by means of a matrix $\mathbf{G}$ of mode coupling factors plus error terms $\mathbf{e}$ :

$$
\mathbf{p}=\mathbf{G a}+\mathbf{e} .
$$

The elements of matrix $\mathbf{G}$ are determined from (1). The optimum estimate for the mode amplitudes that minimizes the sum of squared errors $\mathbf{e}^{H} \mathbf{e}=(\mathbf{a}-\hat{\mathbf{a}})^{H}(\mathbf{a}-\hat{\mathbf{a}})$ at the sensors is given by

$$
\hat{\mathbf{a}}=\mathbf{G}^{+} \mathbf{p}
$$

where $\mathbf{G}^{+}=\left(\mathbf{G}^{H} \mathbf{G}\right)^{-1} \mathbf{G}^{H}$ denotes the pseudo inverse of $\mathbf{G}$. Of interest in broadband noise is the cross spectrum of mode amplitudes given by

$$
\mathbf{S}_{a a}=\lim _{T \rightarrow \infty} E\left\{\frac{1}{T} \hat{\mathbf{a}} \hat{\mathbf{a}}^{H}\right\}=\lim _{T \rightarrow \infty} E\left(\begin{array}{cccccc}
\left\{a_{1} a_{1}^{*}\right\} & \left\{a_{1} a_{2}^{*}\right\} & \left\{a_{1} a_{3}^{*}\right\} & \cdot & \cdot & \left\{a_{1} a_{L}^{*}\right\} \\
\left\{a_{2} a_{1}^{*}\right\} & \left\{a_{2} a_{2}^{*}\right\} & \cdot & \cdot & \cdot & \left\{a_{2} a_{L}^{*}\right\} \\
\cdot & \cdot & \left\{a_{3} a_{3}^{*}\right\} & \cdot & \cdot & \cdot \\
\cdot & \cdot & \cdot & \cdot & \cdot & \cdot \\
\cdot & \cdot & \cdot & \cdot & \cdot & \cdot \\
\left\{a_{K} a_{1}^{*}\right\} & \cdot & . & \cdot & \cdot & \left\{a_{K} a_{L}^{*}\right\}
\end{array}\right) .
$$

Substituting (4) into (5) gives

$$
\mathbf{S}_{a a}=\lim _{T \rightarrow \infty} E\left\{\frac{1}{T} \mathbf{G}^{+} \mathbf{p} \mathbf{p}^{H}\left(\mathbf{G}^{+}\right)^{H}\right\}
$$




$$
\mathbf{S}_{a a}=\mathbf{G}^{+} \mathbf{S}_{p p}\left(\mathbf{G}^{+}\right)^{H}
$$

where

$$
\mathbf{S}_{p p}=\lim _{T \rightarrow \infty} E\left\{\frac{1}{T} \mathbf{p p}^{H}\right\} .
$$

From the measured cross spectrum of mode amplitudes the mode coherence function between two modes $(m, n)$ and $(\mu, \nu)$ can be calculated as

$$
C_{m n}^{\mu \nu}=\frac{\left|\left\langle A_{m n} A_{\mu \nu}^{*}\right\rangle\right|^{2}}{\left\langle\left|A_{m n}\right|^{2}\right\rangle\left\langle\left|A_{\mu \nu}\right|^{2}\right\rangle}
$$

where $A_{m n}$ and $A_{\mu \nu}$ denote the amplitudes deduced from (7). Note that $A_{m n}$ (and $A_{\mu \nu}$ ) represent statistical quantities in this context, whereas the same symbol represents a deterministic quantity in (1).

\section{II.B. Mode coherences for point sources}

If individual point sources in a duct are considered, the method of Green's function can be used to determine the resulting sound field and the resulting coherences between different propagating modes. The details of the theory will be explained elsewhere in more detail, while here only those results will be presented that are necessary for the comparison with the experimental data in sections III and IV.

The number of random monopole sources is denoted by $J$. It is assumed that these sources share the same source spectrum and the same radial coordinate, but they can exhibit different axial positions $x_{j}$ and azimuthal angles $\phi_{j}$. If the point sources are mutually uncorrelated, the coherence between two waves $(m, n)$ and $(\mu, \nu)$ propagating in the positive $x$-direction is found to be

$$
C_{m n}^{\mu \nu}(\omega)=\frac{1}{J^{2}}\left|\sum_{j=1}^{J} e^{i(m-\mu) \phi_{j}} e^{i\left(k_{m n}^{+}-k_{\mu \nu}^{+}\right) x_{j}}\right|^{2} .
$$

If all sources exhibit the same axial coordinate, i.e., they lie in a single ring, and if they are placed equidistantly in the azimuthal direction, then (10) yields a particularly simple result: The coherence is 0 if the azimuthal difference $m-\mu$ is not an integer multiple of $J$, while the coherence is 1 , if $m-\mu i s$ an integer multiple of $J$. This result holds for all frequencies $\omega$ and corroborates Ref. ${ }^{3}$

Finally, two rings of sources are considered. They are located at $x_{1}$ and $x_{2}$, respectively. The case is studied where both rings exhibit the same number $J_{0}$ of sources and where the sources in both rings are distributed equidistantly in the azimuthal direction. Two distinct cases concerning the relative orientation of the two rings are considered: the parallel configuration where the sources in both rings exhibit the same azimuthal positions, and the staggered configuration where the sources in one ring lie in the middle between the sources of the other ring (with respect to $\phi$ ), i. e., one ring is rotated with respect to the other by the angle $\frac{1}{2}\left(2 \pi / J_{0}\right)$. For these two configurations, the coherence is 0 if $m-\mu$ is not an integral multiple of $J_{0}$. If, however, $m-\mu=l J_{0}$ for some integer $l$, then the coherence is given by

$$
C_{m n}^{\mu \nu}(\omega)=\frac{1}{2}\left[1 \pm \cos \left(\left(k_{m n}^{+}-k_{\mu \nu}^{+}\right)\left(x_{2}-x_{1}\right)\right)\right] .
$$

In case of the parallel configuration, only the plus sign is present, while for the staggered configuration the plus sign relates to $l$ being even and the minus sign to $l$ being odd. The latter case can be used to discriminate the two configurations since different coherences result.

\section{Basic test setup S1}

\section{III.A. Overview of experiment}

For the validation of the broadband mode analysis method, DLR conducted experiments in a DLR acoustic test rig, which allowed for testing under well-defined conditions. The setup is depicted in Figure 1 and was described in detail in Ref. ${ }^{1}$ In the following a brief overview of the key elements is given. Broadband sound fields of different modal signatures and coherences were generated using a wall-flush mounted loudspeaker 


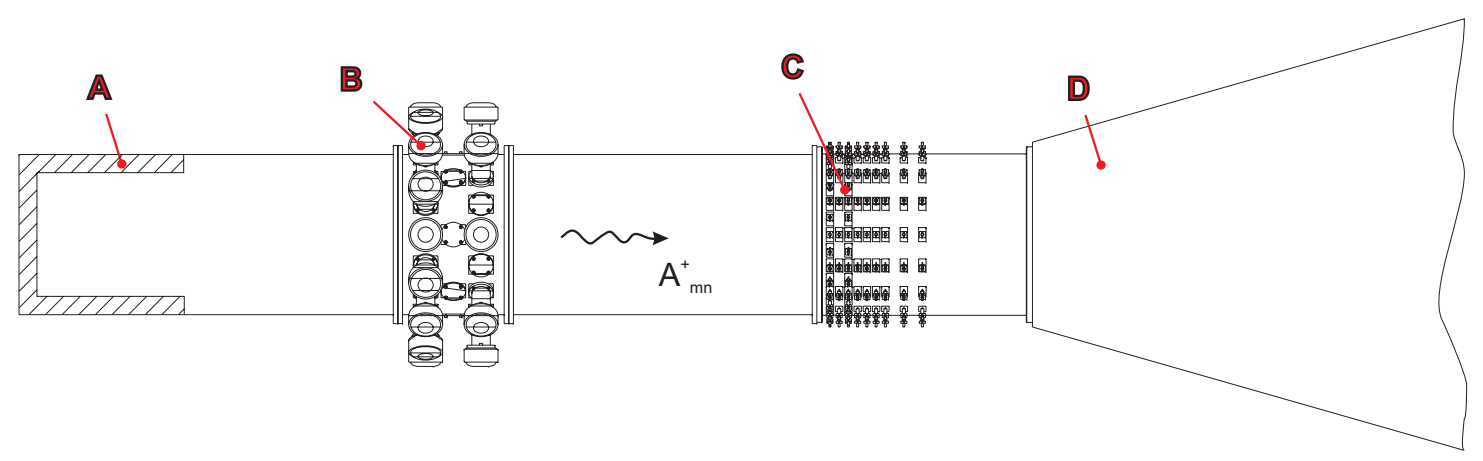

Figure 1. DLR acoustic test rig in setup S1 (no-flow configuration) used for validation of the mode analysis method. Broadband sound fields of different modal signatures and coherences were generated with help of the loudspeaker array $B$ and measured using the array of microphones C. The duct was terminated at both ends by non-reflecting terminations $\mathrm{A}$ and $\mathrm{D}$.

array. In the array up to 16 out of 24 loudspeakers arranged in one or two rings were fed with independent random signals from a digital signal generator board conditioned for the frequency range $800 \mathrm{~Hz}$ to $20000 \mathrm{~Hz}$. Compression drivers were used in combination with conical horns as loudspeaker units. It should be noted that due to the conical horn between each compression driver and the duct wall, mode scattering is inherent to the individual measurements. For the frequency range analyzed in this paper $k r_{l} \ll 1$ was satisfied (with $r_{l}$ being the radius of the loudspeaker opening at the duct wall), i. e., the loudspeakers were well approximating point-like monopole sources. The inner radius of the rig duct was $R=250 \mathrm{~mm}$.

The resulting sound field was acquired by means of a wall-flush mounted microphone array consisting of 96 microphones arranged in 6 rings. The microphone array was designed such that radial mode decomposition was feasible up to a frequency of $2.1 \mathrm{kHz},{ }^{5}$ corresponding to $k R=9.6, \max (m)=7$ and $\max (n)=2$. The acquired time series were Fourier transformed with a frequency bandwidth of approximately $2 \mathrm{~Hz}$ in order to establish the spectral cross correlation matrix for each narrow-band frequency component needed as input for the analysis method.

\section{III.B. Results for a single ring of sources}

The first test case consists of a single ring of 8 equally spaced loudspeakers in duct section $\mathrm{B}$. The resulting coherences between the various propagating modes were determined by applying the method described in section II.A to the microphone signals measured in duct section C. Only results for modes traveling to the right are reported here. Up to $k R=9.6$, a total number of 27 waves can propagate in the duct resulting in $\left(27^{2}-27\right) / 2=351$ non-trivial combinations between two modes and thus the same number of coherences. In order to get an overview over this large number of coherences, they are sorted according to the results from the theory outlined in section II.B. According to the paragraph following equation (10), the coherence is expected to be 0 for all frequencies if the azimuthal difference $m-\mu$ of the two waves $(m, n)$ and $(\mu, \nu)$ is not an integer multiple of the number of sources (8). This condition is satisfied by a total number of 318 mode pairs and their coherence is shown in Figure 2(a). If, in contrast, $m-\mu$ is an integer multiple of 8 , then the coherence is 1 according to the theory. There are 33 mode pairs satisfying this condition and their coherence is depicted in Figure 2(b).

Generally a good agreement between theory and experiment is observed. Deviations occur mainly in the form of isolated spikes near the cut-off frequencies of waves. These deviations can be attributed to numerical difficulties in the determination of the modal composition. Apart from the spikes, the coherence is found to increase with increasing frequency in Figure 2(a). This might be explained by the fact that the number of possible mode combinations increases drastically with increasing frequency and deviations for certain mode pairs become more and more likely.

In Figure 2(b) - apart from spikes - only the three mode pairings between the waves $(0,0),(0,1)$, and $(0,2)$ have significantly low coherence in a broad frequency range. This deviation from the theoretical value 1 could be explained by the fact that it is difficult to distinguish these modes with a wall-mounted array at high frequencies. In addition, it might be that waves which are reflected in the termination A interfere with the primary sound field propagating from B to C. 
(a)

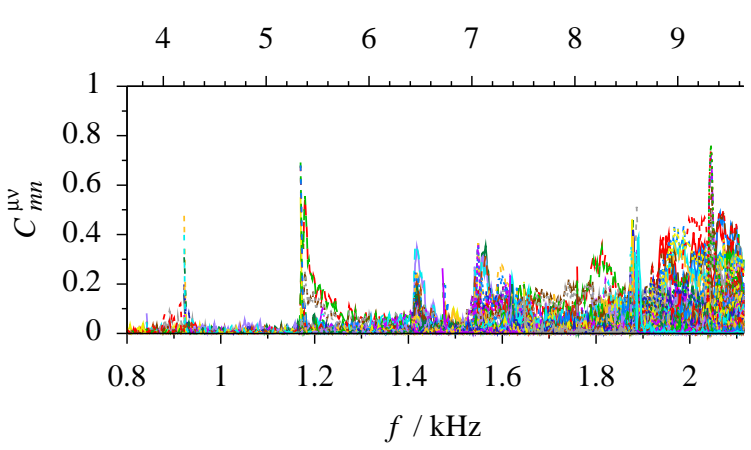

(b)

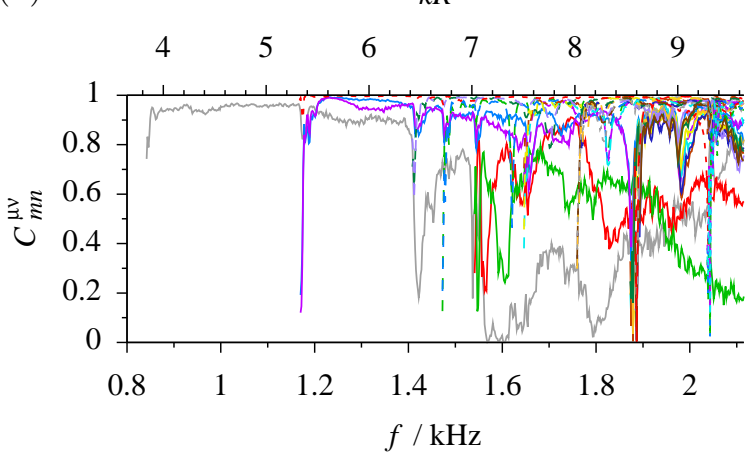

Figure 2. Mode coherence functions obtained for a single ring of 8 equally spaced incoherent sources. Diagram (a) depicts coherences for mode pairs with azimuthal difference $m-\mu$ not being an integer multiple of 8 . Diagram (b) shows coherences for all mode pairs with $m-\mu$ being an integer multiple of 8 . In (b), the curve for $C_{0,0}^{0,1}$ is colored in gray, that for $C_{0,0}^{0,2}$ in red, and that for $C_{0,1}^{0,2}$ in green.

\section{III.C. Results for two rings of sources}

In this section the results obtained from the theory in section II.B are compared with experimental findings for the case of two rings of sources. For this purpose, the duct section B was equipped with two rings of loudspeakers which exhibit a distance of $180 \mathrm{~mm}$. Each ring comprises 8 individual speakers which are distributed equidistantly around the circumference.

Two different configurations were investigated: the parallel and the staggered configuration as explained in section II.B. Mode coherences can be used to discriminate the two configurations if mode pairs are considered with azimuthal difference $m-\mu$ equal to an odd multiple of the number of loudspeakers in one ring. The coherences for two such mode pairs, $(+4,0),(-4,0)$ and $(+3,0),(-5,0)$, are shown in Figure 3. Theoretical curves from (11) are added in the right diagrams, while for the left diagrams equation (11) yields 1 for the parallel configuration (Figure 3a) and 0 for the staggered configuration (Figure 3c). Theory and experiment match well in the left diagrams, whereas deviations occur in the right diagrams. These deviations might be caused by reflections in the termination A.

Despite the deviations, the comparison reveals that particularly the mode pair $(+4,0),(-4,0)$ is suited to identify the type of source configuration that is present in the duct. This observation underlines the potential of the investigation of mode coherences.

\section{Test setup S2 simulating acoustic features of turbo-shaft engines}

\section{IV.A. Overview of experiment}

In Figure 4 the DLR acoustic test rig is shown in an extended version S2 with flow. It was designed with the intention to capture the most relevant acoustic features of a turbo-shaft engine in the low to medium frequency range. In particular, the superposition of combustion noise with turbine broadband noise can be studied with this test rig, as well as propagation effects such as mode and frequency scattering. In order to enable a comparison with theoretical results, the setup was kept as simple as possible.

In the experiment the loudspeaker array B served as a combustion noise simulator producing test sound fields with similar spectral characteristics, mode content, and mode correlations as described above for the generic test setup S1. For the investigation of frequency scattering effects further tests with band-pass filtered noise were carried out. ${ }^{6}$

The sound field emitted by the simulated combustor noise source $\left(A_{m n}^{+}\right)$was measured with help of the microphone array $\mathrm{C}$ located between the loudspeaker array and the axial fan. A fan was introduced in reverse direction as simplest means to represent a single stator-rotor stage of a turbo-shaft engine turbine - not in the aerodynamic sense, but with regard to the acoustic scattering at the vanes and blades. The fan featured $B=24$ rotor blades and $V=5$ strut-like vanes, all without aerodynamic shape and of radially straight form. The mode content reflected at the stator-rotor stage $\left(A_{m n}^{-}\right)$was measured using microphone array $\mathrm{C}$, the modes transmitted through the stage $\left(B_{m n}^{+}\right)$were measured with microphone array $\mathrm{E}$. Array $\mathrm{C}$ was equipped with four rings, each holding 16 microphones so that frequencies up to $1.5 \mathrm{kHz}$ corresponding 
(a)

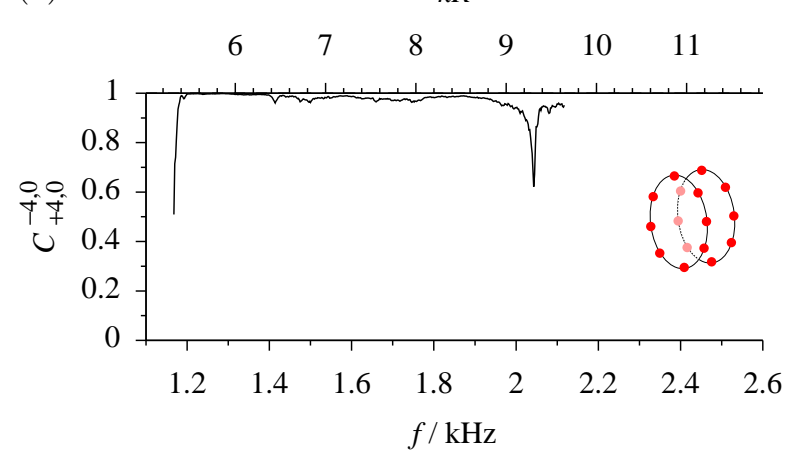

(c)

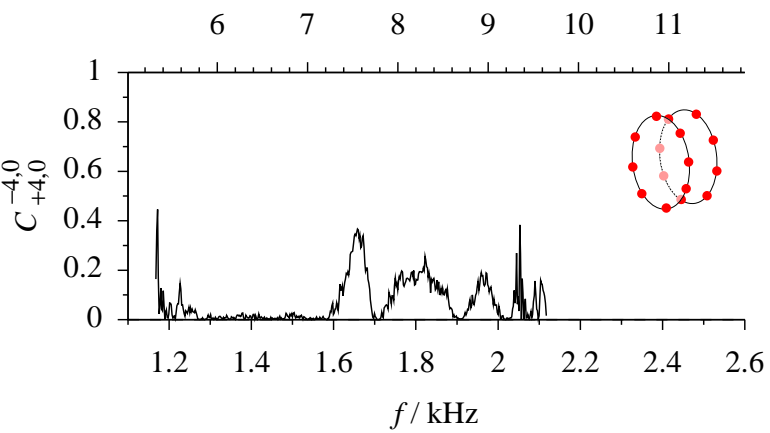

(b)

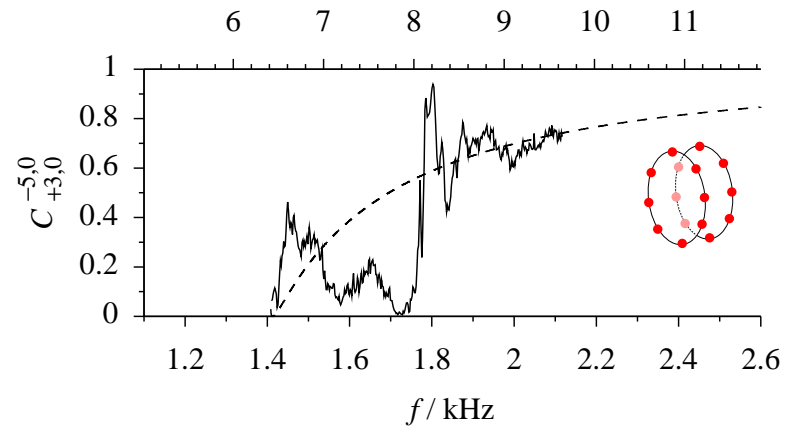

(d)

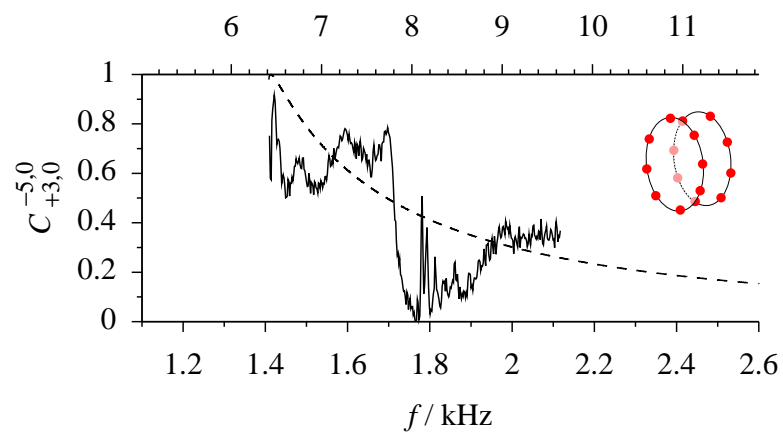

Figure 3. Mode coherences obtained for two source rings, each with 8 incoherent sources. In the top diagrams (a,b) the source rings were parallel (azimuthally not staggered), while in the bottom diagrams (c,d) the source rings were azimuthally staggered by an angle $2 \pi / 16$. The left diagrams (a,c) show the mode coherence measured for mode pairing $(+4,0)$ and $(-4,0)$. In the right diagrams $(\mathbf{b}, \mathbf{d})$ the measured mode coherences $(-)$ are compared with the analytical solutions $(--)$ for mode pairing $(+3,0)$ and $(-5,0)$.

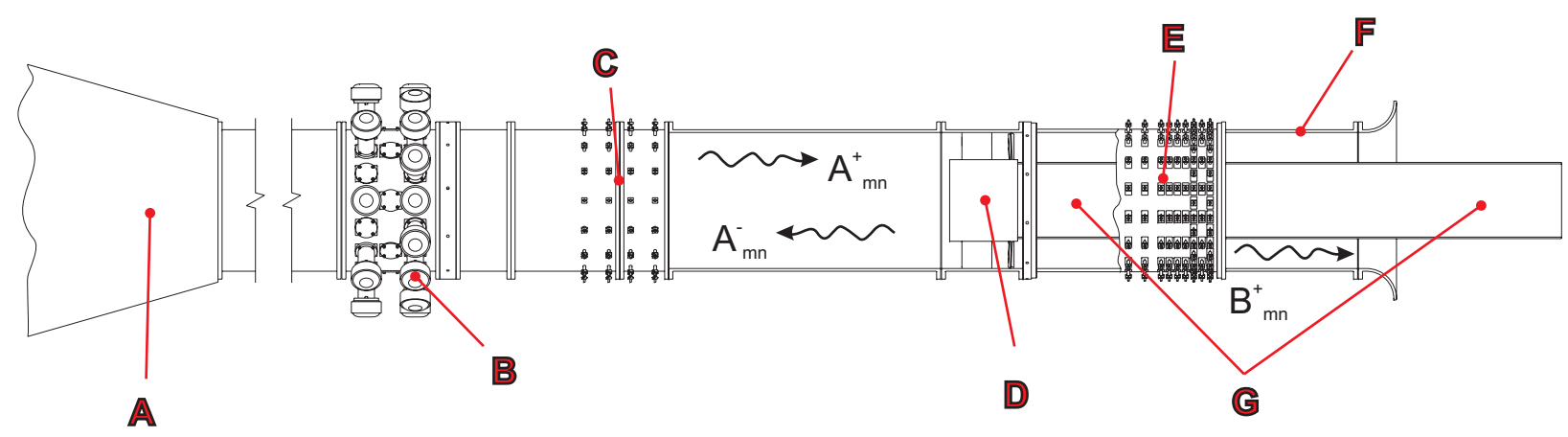

Figure 4. DLR acoustic test rig prepared in setup S2 for investigation of the most relevant acoustic features of a turboshaft engine. The loudspeaker array $B$ served as a combustor noise source simulator, an axial fan $D$ represented a stator-rotor stage for the investigation of mode scattering effects. The emitted, reflected and transmitted modal sound field components were measured by sensor arrays $C$ and $E$, respectively. The rig was terminated with an anechoic termination at one side and a nozzle at the opposite side. 
to $k R=6.9, \max (m)=5$, and $\max (n)=1$ could be analyzed.

Microphone array E attached to the stator-rotor stage consisted of 80 wall-flush mounted microphones in 4 rings. Three rings each with 16 microphones were equally spaced in circumferential direction in a distance of $545 \mathrm{~mm}, 605 \mathrm{~mm}$, and $665 \mathrm{~mm}$ to the rotor. The fourth ring consisted of 32 microphones in a distance of $725 \mathrm{~mm}$ to the rotor. It is possible to analyze frequencies up to $3.1 \mathrm{kHz}$ with array E corresponding to $k R=14.2, \max (m)=12$, and $\max (n)=1$.

It should be noted that a hub was introduced upstream of the fan. Despite the fact that the flow conditions - and consequently the variation of the mode cut-off ratios - were not representative, the hub modified the conditions for mode propagation and thus allowed a more differentiated verification of the employed broadband noise analysis method.

\section{IV.B. Results: Mode amplitude spectra}

Figure 5 shows mode amplitudes $A_{m n}^{+}$in relative units for modes propagating from left to right. (Note that the notation $(m, n)+$ and $(m, n)$ - is used in this and the following section IV.C in order to describe the propagation direction of a mode.) The amplitudes of the modes were measured in array $\mathrm{C}$ using (7) with a frequency resolution of approximately $8 \mathrm{~Hz}$. In Figure 5(a) only the rotor is operating with a rotation frequency of $25 \mathrm{~Hz}$ (=1500 rpm) whereas no loudspeakers were used. In Figure 5(b) the mode amplitudes are shown for the case of a stationary rotor while 8 loudspeakers mounted equidistantly in a single ring in duct section B were driven with incoherent signals. Figure 5(c) depicts the amplitudes for the combination of both cases: the rotor turns with $25 \mathrm{~Hz}$ and 8 loudspeakers operate. This situation models the case where a dominant combustion noise (modeled by the loudspeakers) propagates through a stator-rotor stage. The transmitted mode amplitudes $B_{m n}^{+}$propagating from left to right are measured in array E, see Figure 6 .

The comparison between mode amplitudes in array $\mathrm{C}$ and $\mathrm{E}$ is complicated by the fact that the installed hub changes the axial wavenumbers and cut-off frequencies of the waves. Nevertheless a comparison of the amplitudes for individual modes is tried in what follows. According to Figure 5(b,c) and $6(\mathrm{~b}, \mathrm{c})$, the amplitudes in array $\mathrm{E}$ are generally lower than in array $\mathrm{C}$ which can be attributed to the shadowing effect of the stator-rotor element.

If the loudspeakers do not operate and only the rotor turns, negligible mode amplitudes are measured in array $\mathrm{C}$ according to Figure 5(a) since only modes propagating in the positive direction are analyzed. These can only emerge from marginal sound reflections at the termination A. In contrast, higher amplitudes are observed in array E according to Figure 6(a), because the direct sound field is measured there.

Finally, it is observed in $5(\mathrm{~b}, \mathrm{c})$ and $6(\mathrm{~b}, \mathrm{c})$ that in the frequency range $1000 \mathrm{~Hz}$ to $1500 \mathrm{~Hz}$ modes with a high amplitude in array $\mathrm{C}$ also exhibit a high amplitude in array $\mathrm{E}$. Thus the relative intensity of the waves is not significantly changed by the transmission through the stator-rotor stage.

\section{IV.C. Results: Mode coherence spectra}

In this section, we investigate the mode coherences in different sections of the duct. Firstly the sound propagation in the present complex setup S2 is compared with the results obtained for the basic setup S1. For this purpose, Figure 7 shows the coherences between mode pairs propagating from the loudspeaker section B to the microphone array C. It is observed that the modes are fully uncorrelated (Figure 7a) or fully correlated (Figure 7b) depending on the azimuthal difference of the considered mode pair. The results agree with the findings in Figure 2 for the basic setup and agree as well with the theory in section II.B.

In what follows, mode coherences are studied for different propagation directions and segments in the duct. In order to simplify the discussion, only pairings are presented between the mode $(+3,0) \pm$ and another mode $(\mu, \nu) \pm$. Figure 8 depicts such mode coherences measured in case of a stationary rotor and 8 loudspeakers in a single ring. In Figure 8(a), the coherence of mode pairs propagating to the right measured in array $\mathrm{C}$ is depicted. This graph thus represents a selection of the curves present in Figure $7(\mathrm{a}, \mathrm{b})$. The only remaining coherent combination of modes in this selection is the mode pair $(+3,0)+,(-5,0)+$.

Coherence pairings in the array $\mathrm{C}$ with different propagation directions $+x$ and $-x$ are shown in Figure $8(\mathrm{~b})$. Note that the first mode represents an incident mode and the second one a mode reflected at the stator-rotor element. The prominent coherence between the incident mode $(-2,0)+$ and the reflected mode $(+3,0)-$ might be explained with mode scattering at the $V=5$ stator vanes. ${ }^{4}$

Finally, coherences of modes transmitted through the stator-rotor stage are investigated in the array $\mathrm{E}$ for both modes propagating in the same $+x$-direction (Figure $8 \mathrm{c}$ ). The mode pairings $(+3,0)+,(-5,0)+$ 
(a)

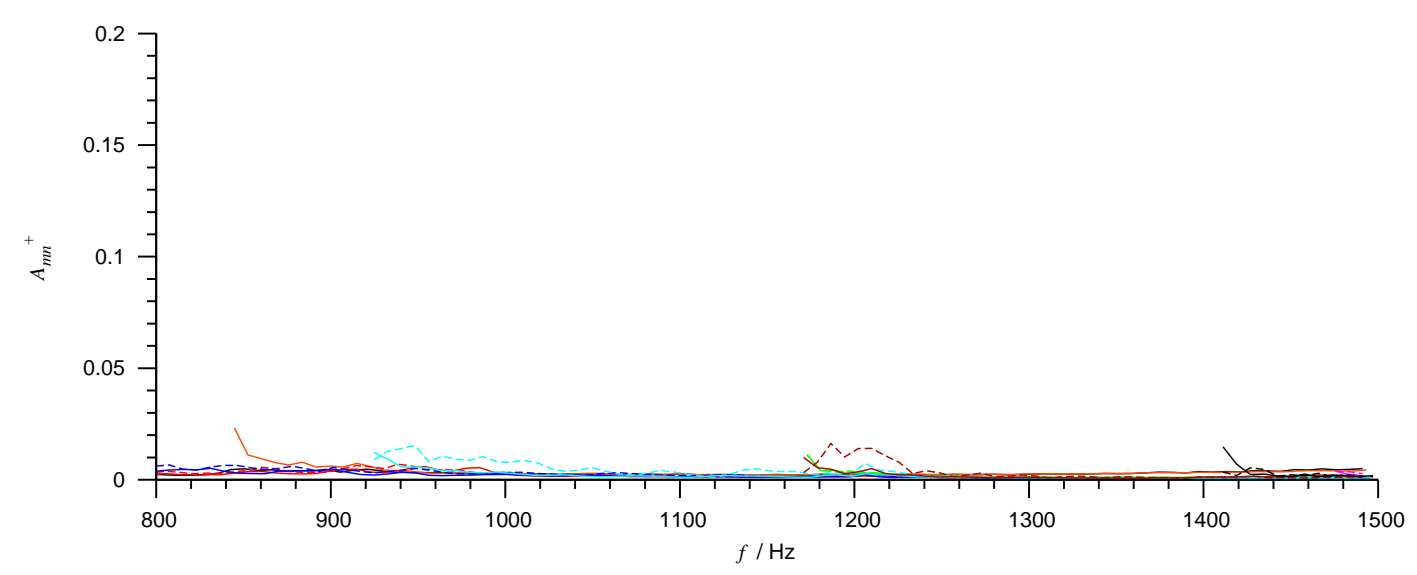

(b)

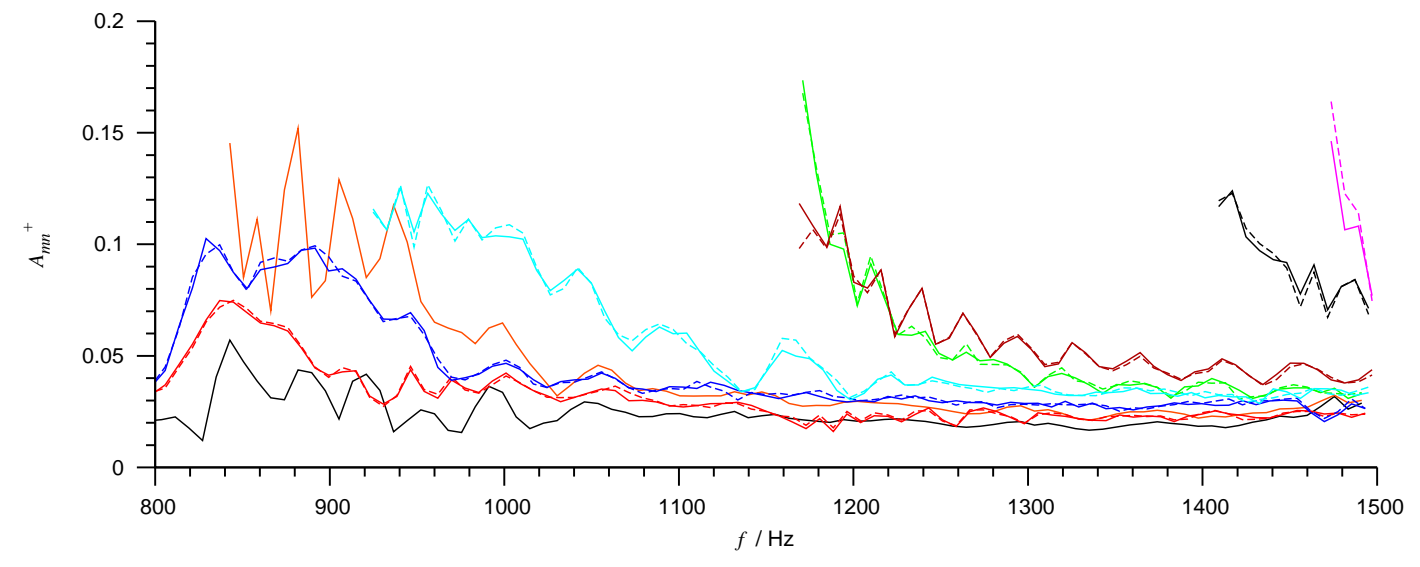

(c)

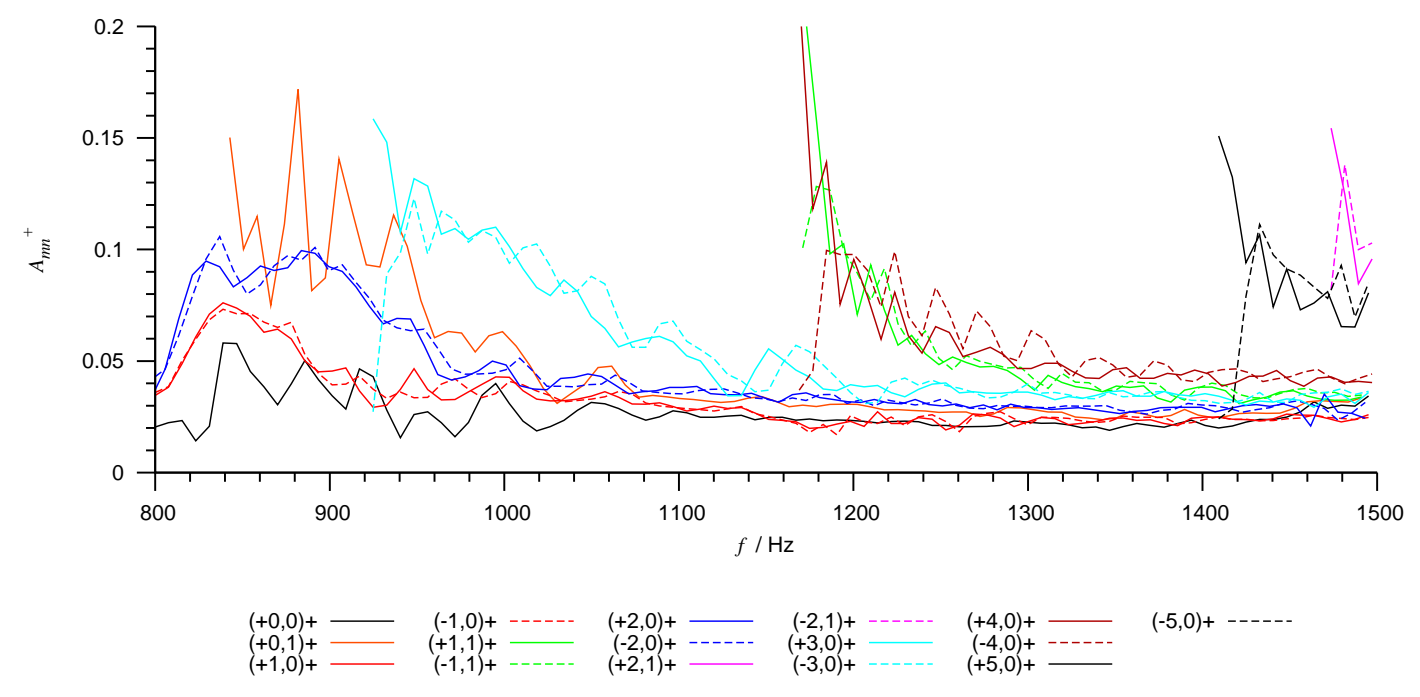

Figure 5. Amplitudes of the modes $A_{m n}^{+}$propagating from left to right and measured with sensor array C. The spectra were measured for: (a) no loudspeakers and rotor turning with $25 \mathrm{~Hz}$, (b) eight loudspeakers and stationary rotor, (c) eight loudspeakers and rotor turning with $25 \mathrm{~Hz}$. 
(a)

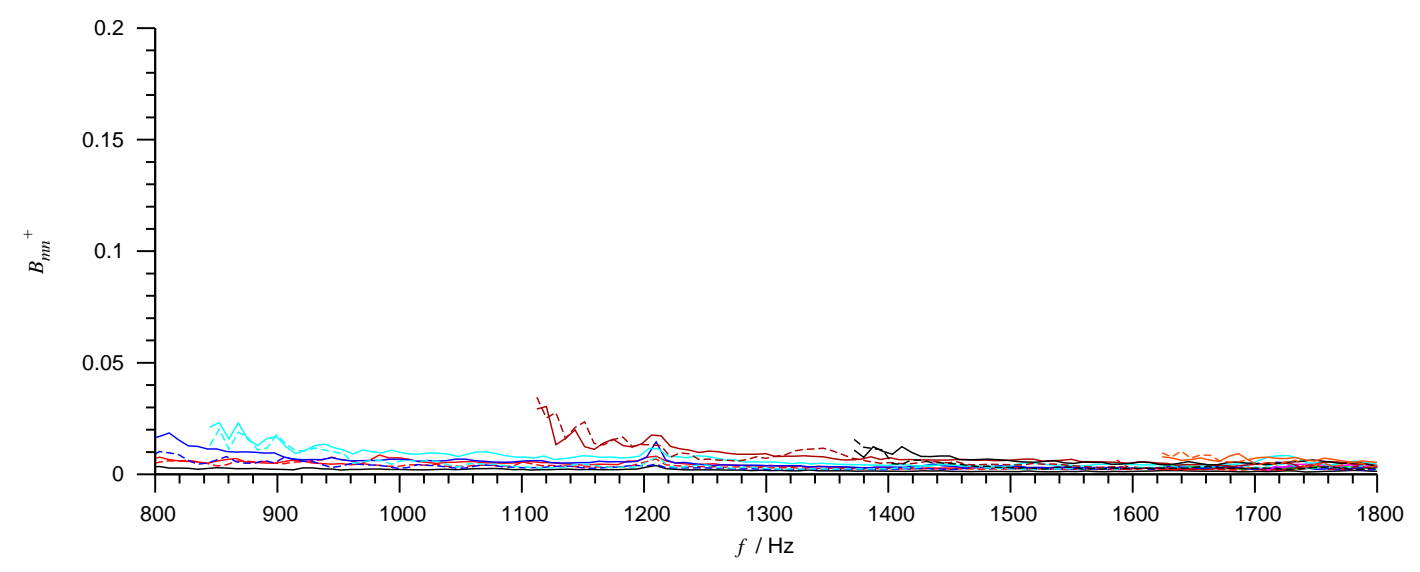

(b)

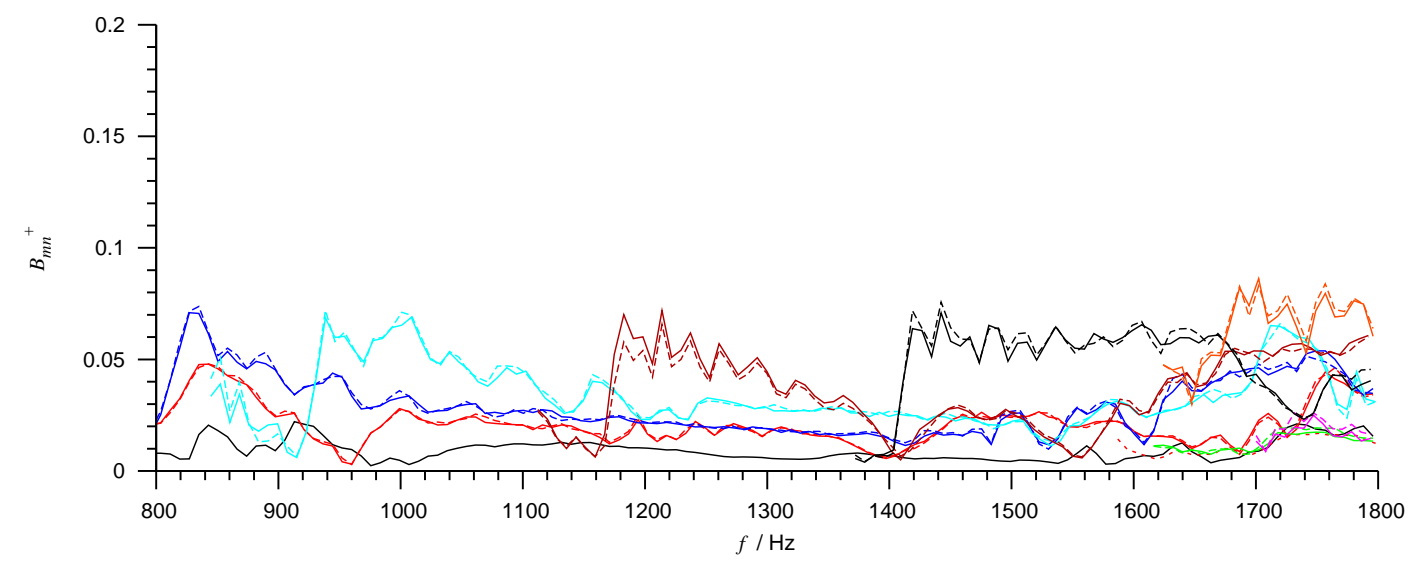

(c)

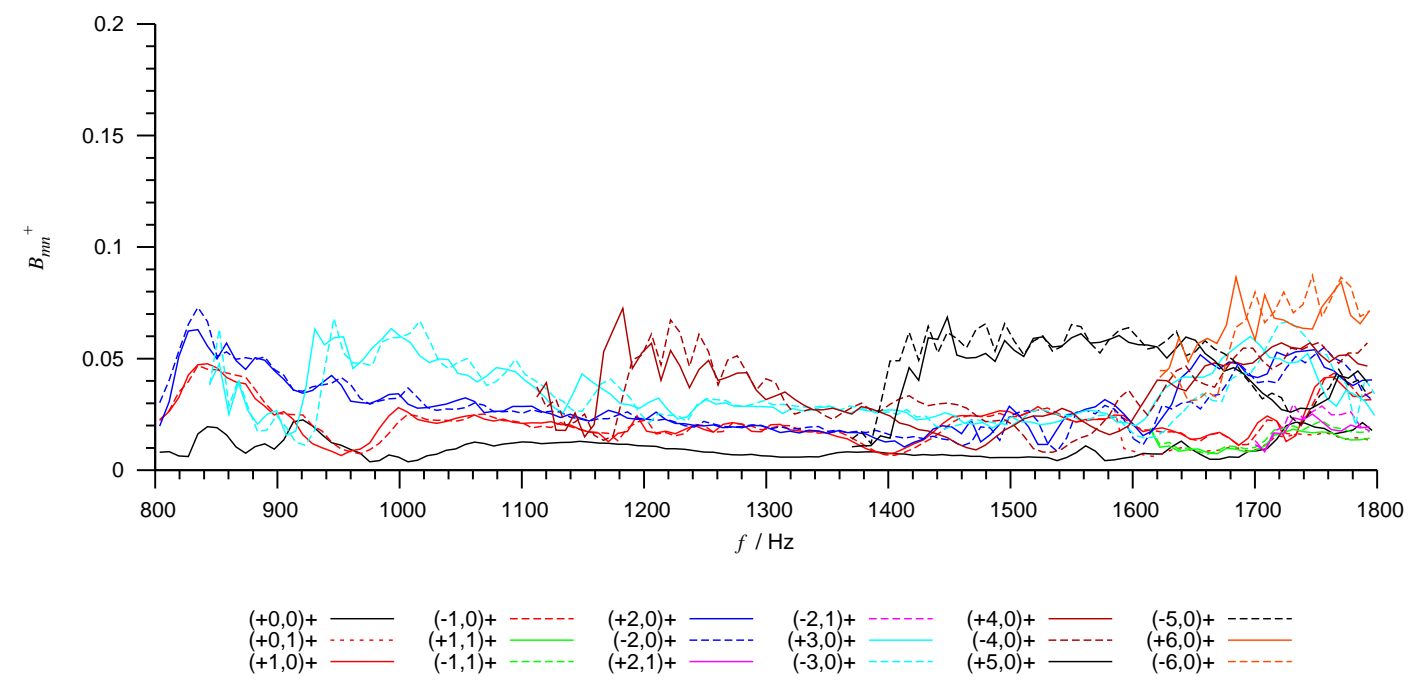

Figure 6. Amplitudes of the modes $B_{m n}^{+}$measured in array E. The three graphs relate to the same cases as in Figure 5 . 
(a)

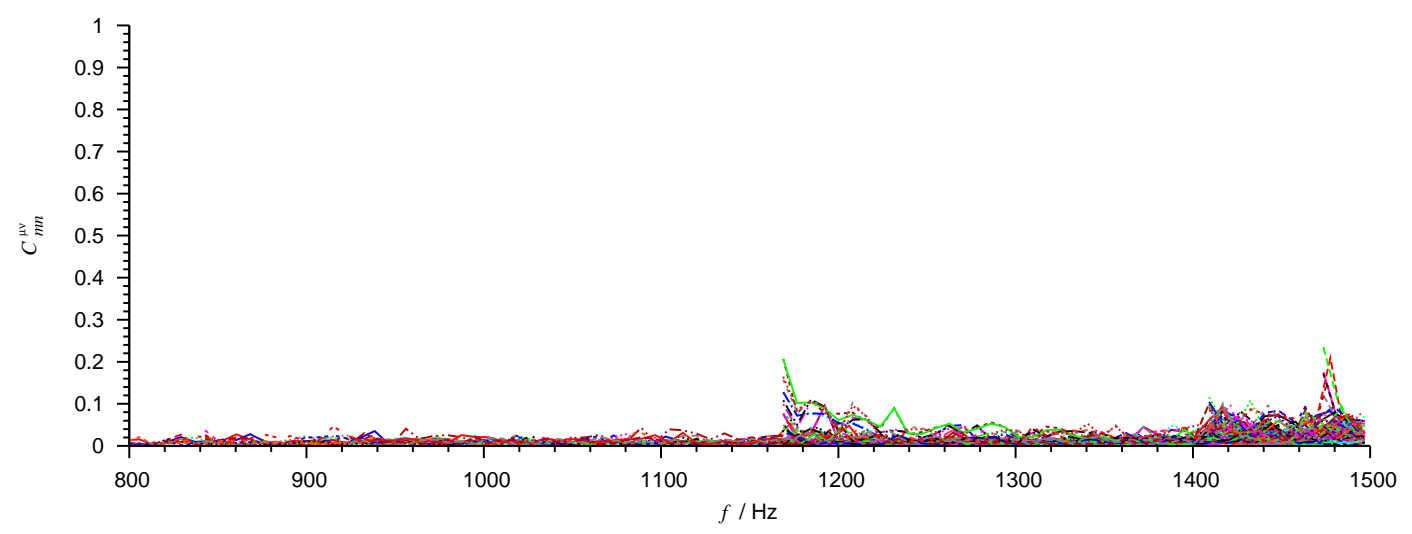

(b)

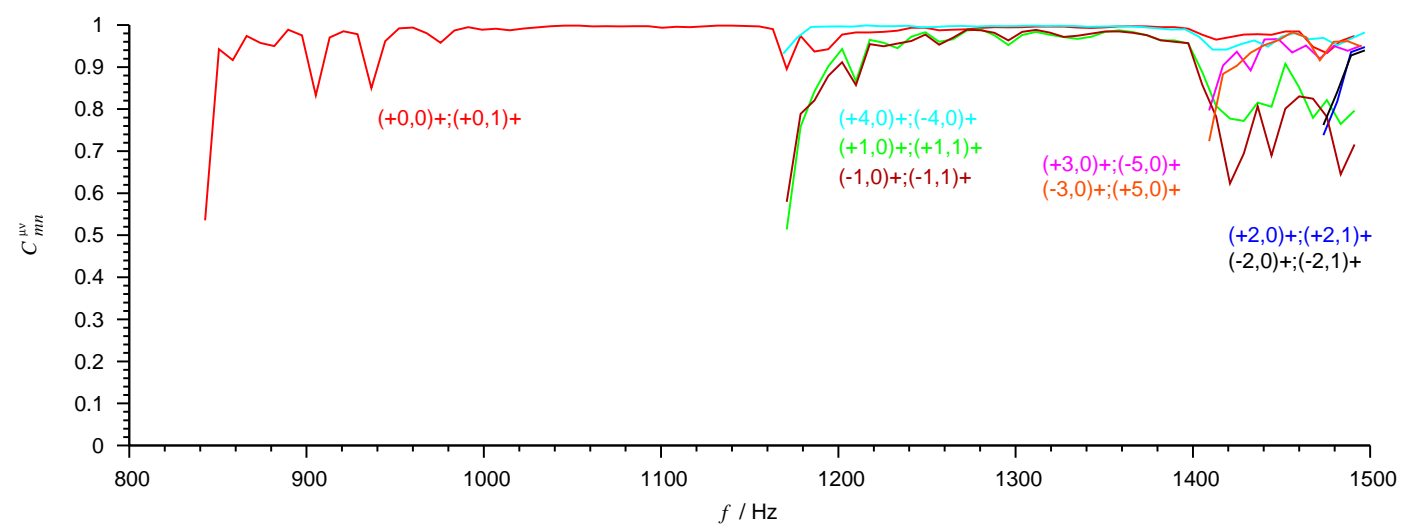

Figure 7. Mode coherences in array $\mathrm{C}$ for a ring of 8 loudspeakers and a stationary rotor. (a) Coherence for modes with azimuthal difference not equal to an integer multiple of 8. (b) Coherence for modes with azimuthal difference equal to an integer multiple of 8.

and $(+3,0)+,(+5,0)+$ are found to exhibit a substantial coherence. The first pair $(+3,0)+,(-5,0)+$ yields coherence 1 in section $\mathrm{C}$ according to Figure 8(a). The reduced coherence for this pair in Figure 8(c) could be caused by the cross-sectional jump or by the characteristic influence of the stator-rotor element. The coherence for the second pair $(+3,0)+,(+5,0)+$ in Figure $8(\mathrm{c})$ might be explained by the fact that the incident mode $(-5,0)+$ is scattered to $(+5,0)+$ while passing the 5 stator vanes. However, no coherence is observed for the pairing $(+3,0)+,(0,0)+$. Consequently, further investigations of the scattering process are necessary.

In order to analyze how the rotor influences the modal coherences, Figure 9 depicts the same cases as in Figure 8 , but with a turning rotor. The comparison of figure 8 and 9 reveals that the influence of the rotor on the mode coherences is quite small except for certain mode pairs (for example $(+3,0)+,(-3,0)$ - in Figures $8 \mathrm{~b}$ and $9 \mathrm{~b})$. Besides these differences, the mode coherence spectra are similar. The following important conclusion can thus be drawn: If combustion noise (here the loudspeaker array) is dominant compared with the rotor noise, then the characteristics of the sources can still be detected in the exhaust array.

\section{Conclusion and outlook}

A new broadband noise analysis technique based on mode coherences was introduced and successfully applied to two different experimental setups of laboratory scale. Both setups featured arrays of sources distributed equally over the circumference of a duct. The measured mode coherences for the basic setup S1 were successfully validated against analytical results. The complex setup S2 represented a fundamental model of an aero-engine exhaust duct. Using the broadband noise analysis technique, physical insight was gained concerning the propagation and scattering of broadband sound fields. 
(a)

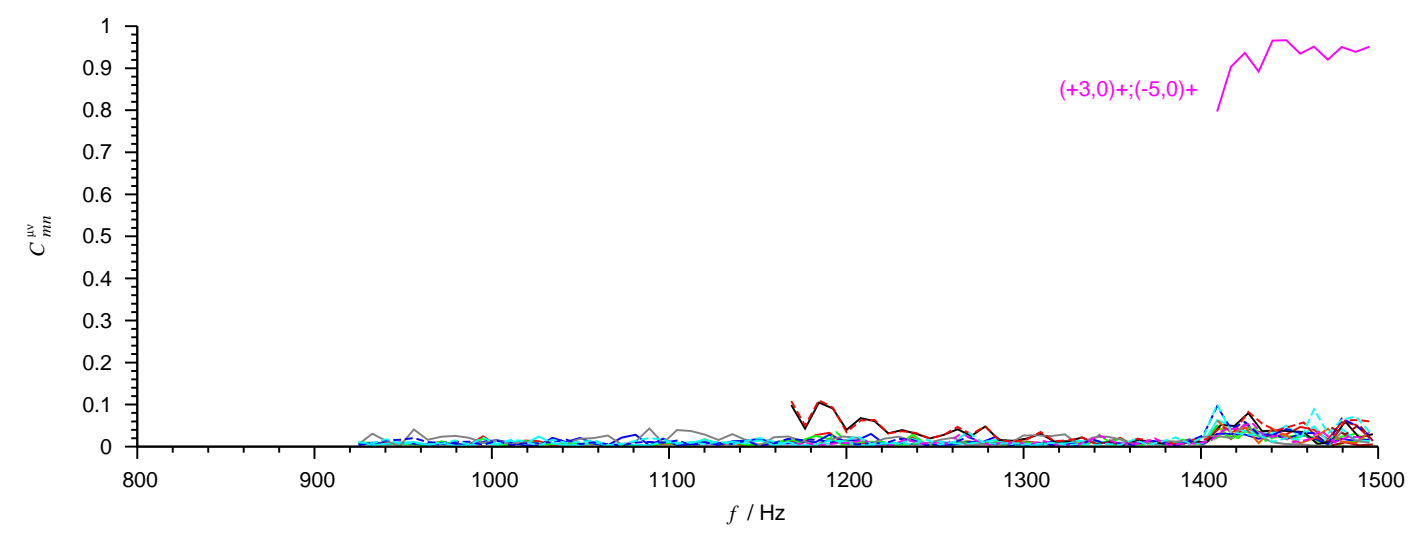

(b)

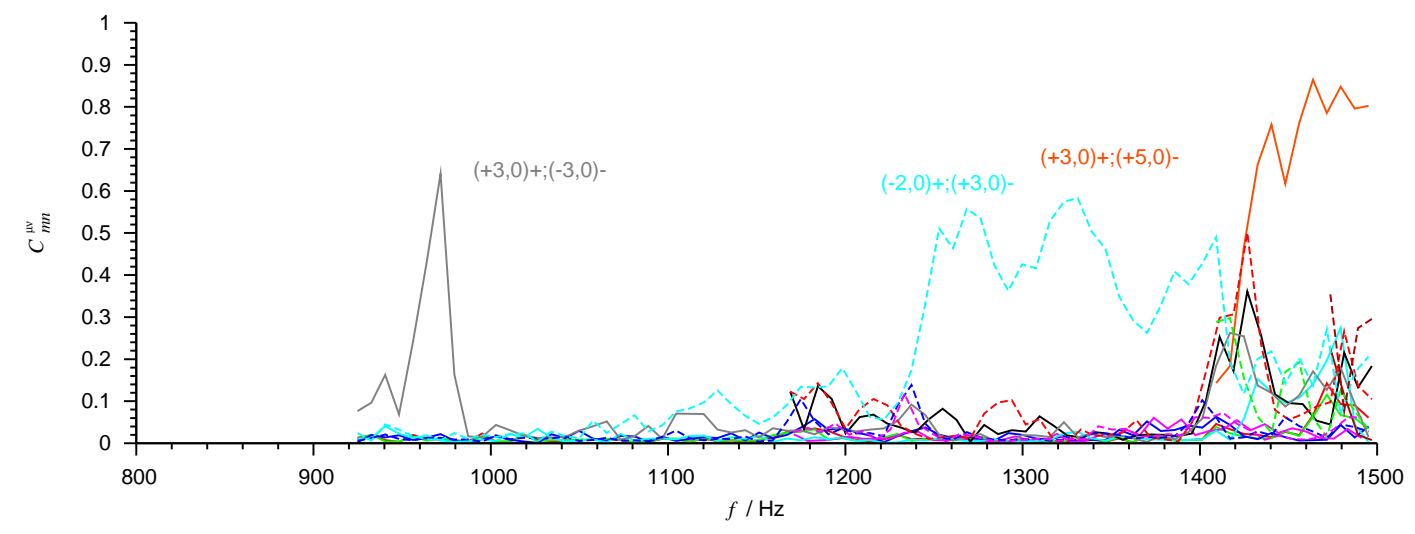

(c)

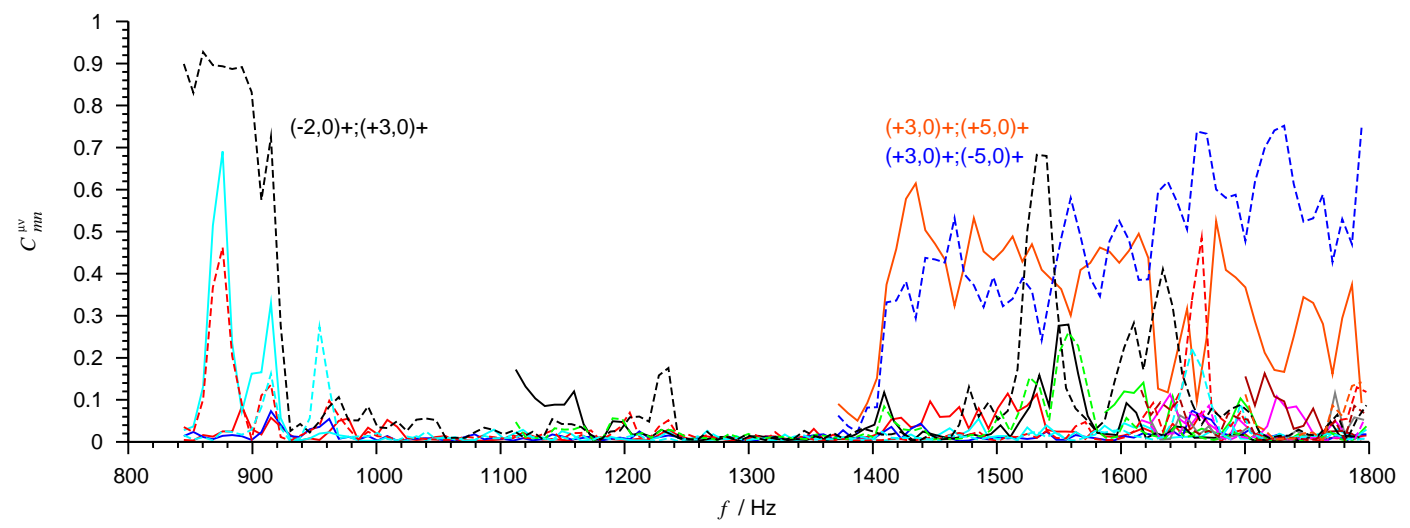

Figure 8. Selected mode coherences measured for a stationary rotor and eight loudspeakers. (a) Mode coherences between two waves in array $\mathbf{C}$, both propagating in the $+x$-direction. (b) Mode coherences in array $\mathbf{C}$ for an incident $(+x)$ and a reflected wave $(-x)$. (c) Mode coherences in array $\mathbf{E}$ between two waves, both propagating in the $+x-$ direction. 
(a)

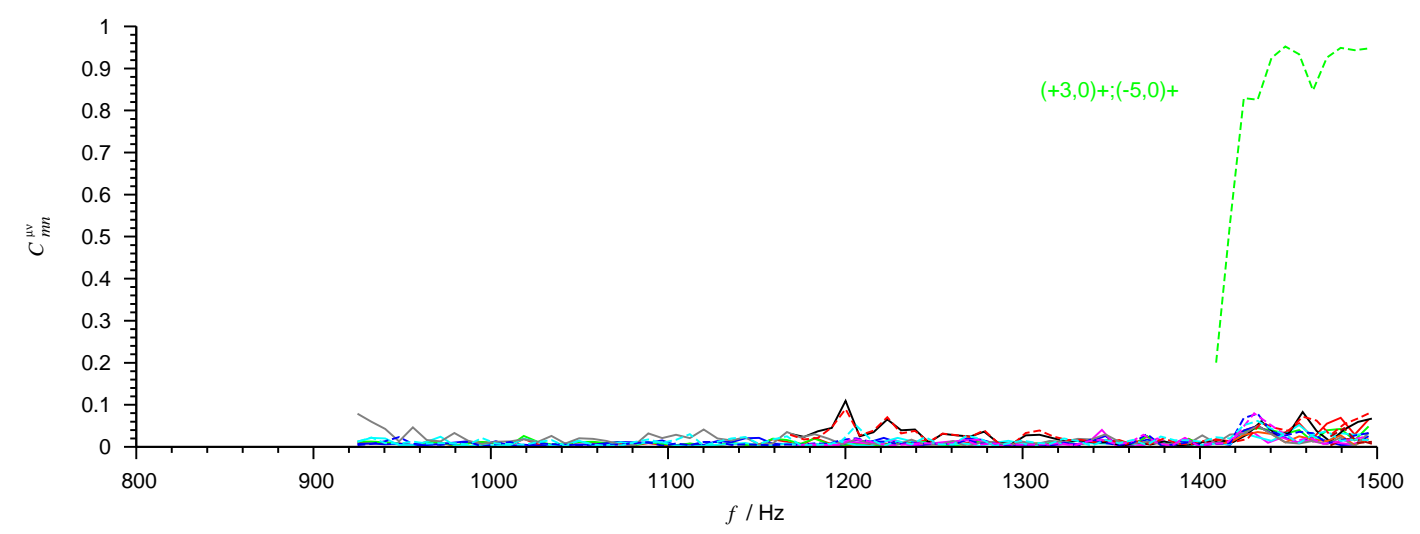

(b)

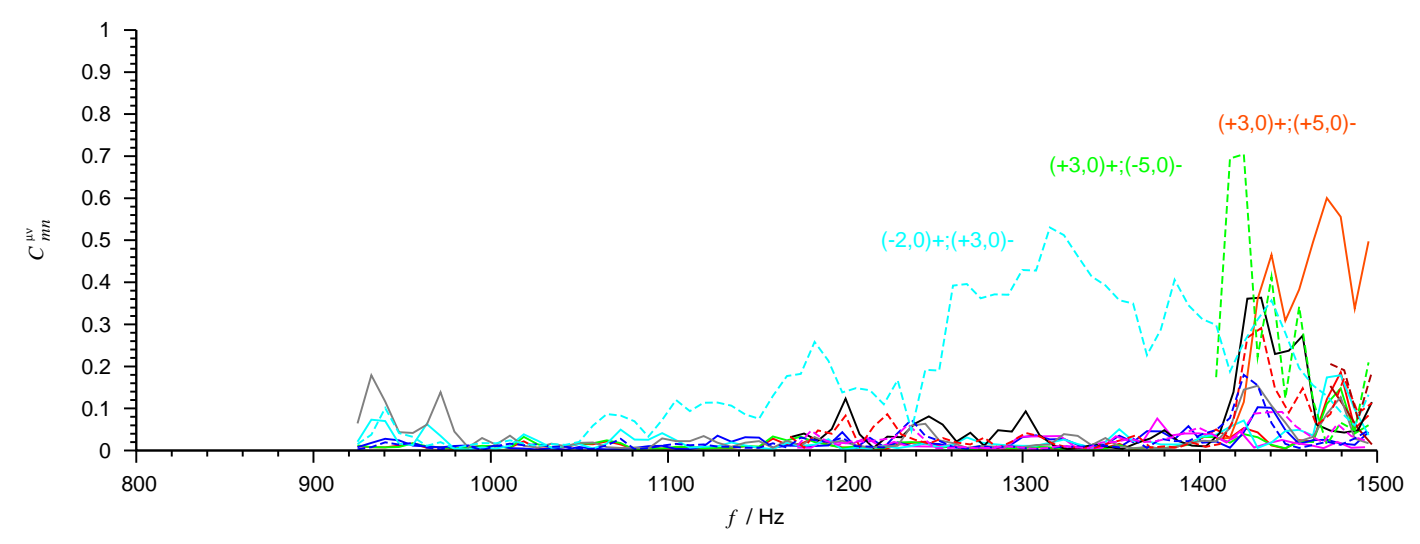

(c)

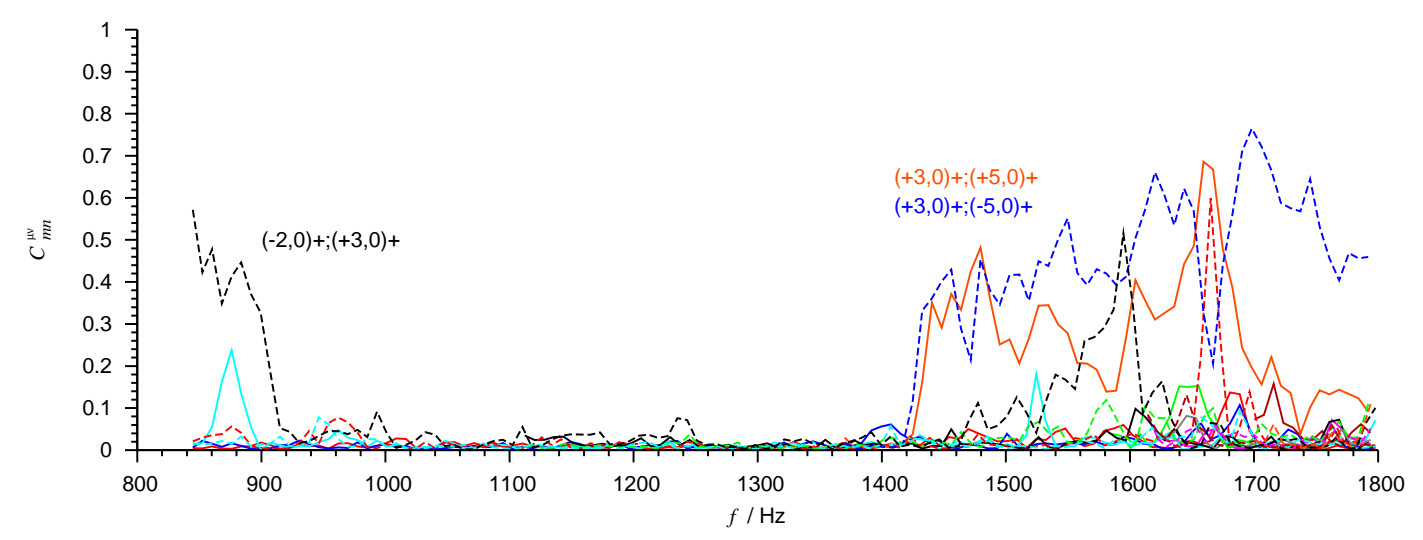

Figure 9. Same as Figure 8 but for the rotor turning with $25 \mathrm{~Hz}$. 
The present investigation, in which sources are spaced equally around the axis, is fundamental for a variety of applications since turbomachinery component designs typically meet symmetry requirements, e. g. in the case of turbine stages or annular combustor arrangements. The measurement of mode coherences can be used to analyze the characteristics of such broadband sources, in particular the number, distribution, and coupling of the individual source elements. In the EU project TEENI, the coherence based acoustical measurement technique will be applied to a full-scale turbo-shaft engine experiment in the near future.

Since the analysis method gives insight into sound source mechanisms, it might aid the development of appropriate noise reduction technologies at the source. Rules and guidelines could even help to better employ active noise control systems dedicated to dominant broadband noise components.

In addition to the analysis of turbomachinery broadband sound fields, the results of the present investigation can also be used to simulate such sound fields by means of loudspeaker arrays. Simulations are useful, for example, for the purpose of studying scattering effects or the radiation into the far field in a laboratory model.

\section{Acknowledgments}

The work presented in this paper was partly supported by the project TEENI (Turbo-shaft Engine Exhaust Noise Identification), which is financed by the European Commission within the 6th Framework Program. P. Joseph from the Institute of Sound and Vibration Research (ISVR), University of Southampton, $\mathrm{UK}$, is acknowledged for contributing to the description of the analysis method (section II.A).

\section{References}

\footnotetext{
${ }^{1}$ Enghardt, L., Holewa, A., and Tapken, U., "Comparison of different analysis techniques to decompose a broad-band ducted sound field in its mode constituents," 13th AIAA/CEAS Aeroacoustic Conference; 21-23 May 2007, Rome, Italy, No. AIAA 2007-3520, 2007.

${ }^{2}$ Michalke, A., Arnold, F., and Holste, F., "On the coherence of the sound field in a circular duct with uniform mean flow," Journal of Sound and Vibration, Vol. 190(2), 1996, pp. 261-271.

${ }^{3}$ Tester, B. J., Sijtsma, P., Joseph, P., and Lowis, C., "Fan broadband noise simulation," 12th AIAA/CEAS Aeroacoustic Conference; 8-10 May 2006, Cambridge, MA, No. AIAA 2006-2684, 2006.

${ }^{4}$ Tyler, J. and Sofrin, T., "Axial Flow Compressor Noise," SAE Transcation, Vol. 70, 1962.

${ }^{5}$ Tapken, U. and Enghardt, L., "Optimization of Sensor Arrays for Radial Mode Analysis in Flow Ducts," 12th AIAA/CEAS Aeroacoustic Conference; 8-10 May 2006, Cambridge, MA, No. AIAA 2006-2638, 2006.

${ }^{6}$ Bennett, G. J., Davis, I., Tapken, U., and Mahon, J., "Non-linear Identification Applied to Broadband Turbomachinery Noise," 16th AIAA/CEAS Aeroacoustic Conference; 7-9 June 2010, Stockholm, Sweden, 2010.
} 